\title{
Possible Benefits of Faecalibacterium prausnitzii for Obesity-Associated Gut Disorders
}

\author{
Tatiani Uceli Maioli ${ }^{1,2 \dagger}$, Esther Borras-Nogues ${ }^{2 \dagger}$, Licia Torres ${ }^{3}$, Sara Candida Barbosa ${ }^{3}$, \\ Vinicius Dantas Martins ${ }^{3}$, Philippe Langella ${ }^{2}$, Vasco Ariston Azevedo ${ }^{4}$ and \\ Jean-Marc Chatel ${ }^{2 \star}$
}

${ }^{1}$ Departamento de Nutrição, Escola de Enfermagem, Universidade Federal de Minas Gerais, Belo Horizonte, Brazil, ${ }^{2}$ Université Paris Saclay, INRAE, AgroParisTech, Micalis, Jouy-en-Josas, France, ${ }^{3}$ Programa de Pós-Graduação em Bioquímica e Imunologia, Instituto de Ciências Biológicas, Universidade Federal de Minas Gerais, Belo Horizonte, Brazil, ${ }^{4}$ Departamento de Genética, Ecologia e Evolução, Instituto de Ciências Biológicas, Universidade Federal de Minas Gerais, Belo Horizonte, Brazil

\section{OPEN ACCESS}

Edited by:

Patricia Machado Rodrigues Silva, Oswaldo Cruz Foundation (FIOCRUZ),

Brazil

Reviewed by:

Ben Woolbright,

University of Kansas Medical Center,

United States

Su Meng,

Hunan University, China

*Correspondence:

Jean-Marc Chatel

jean-marc.chate@inrae.fr

these authors have contributed equally to this work

Specialty section: This article was submitted to Inflammation Pharmacology,

a section of the journal

Frontiers in Pharmacology

Received: 13 July 2021 Accepted: 04 October 2021 Published: 02 December 2021

Citation:

Maioli TU, Borras-Nogues E, Torres L, Barbosa SC, Martins VD, Langella $P$ Azevedo VA and Chatel J-M (2021) Possible Benefits of Faecalibacterium prausnitzii for Obesity-Associated

Gut Disorders.

Front. Pharmacol. 12:740636. doi: 10.3389/fphar.2021.740636
Metabolic disorders are an increasing concern in the industrialized world. Current research has shown a direct link between the composition of the gut microbiota and the pathogenesis of obesity and diabetes. In only a few weeks, an obesity-inducing diet can lead to increased gut permeability and microbial dysbiosis, which contributes to chronic inflammation in the gut and adipose tissues, and to the development of insulin resistance. In this review, we examine the interplay between gut inflammation, insulin resistance, and the gut microbiota, and discuss how some probiotic species can be used to modulate gut homeostasis. We focus primarily on Faecalibacterium prausnitzii, a highly abundant butyrate-producing bacterium that has been proposed both as a biomarker for the development of different gut pathologies and as a potential treatment due to its production of anti-inflammatory metabolites.

Keywords: diabetes, gut permeability, obesity, probiotics, Faecalibacterium prausnitzii

\section{INTRODUCTION}

Obesity has increased worldwide (Di Cesare et al., 2016) and is a public health concern across the globe. This disease is associated health outcomes such as diabetes, hypertension, and cancer (ArroyoJohnson and Mincey, 2016). Currently, it is estimated that $30 \%$ of the global population is overweight, and this number continues to increase (Talukdar et al., 2020). The most prevalent consequence of obesity is insulin resistance and the development of type 2 diabetes (T2D). This condition is driven by inflammation that begins in adipose tissue. The hyperglycemia associated with obesity is linked with gut inflammation, increased permeability to bacterial products, and changes in microbiota composition that promote the cycle of inflammation associated with the obese state (Fallucca et al., 2014). Such pathogenic modifications of the community structure of gut microbiota are referred to as dysbiosis; one of the most well-known examples in the context of obesity is an increase in the ratio of Firmicutes to Bacteriodetes in both mice and humans, which produces a proinflammatory profile characteristic of the obese microbiota (Kim et al., 2012; Verdam et al., 2013).

Diet has a direct influence on intestinal inflammation, which is related to weight gain and microbiota changes. A few weeks of a high-fat, high-sugar diet is enough to induce certain gut disorders, including increased gut permeability and dysbiosis (Cani et al., 2001b). After this it is possible to detect free lipopolysaccharides (LPS) in the blood, which triggers the activation of the innate immune system and inflammatory conditions (Guerville et al., 2017). 
The development of hyperglycemia has been linked with a variety of alterations in the gut mucosa in addition to dysbiosis. These include increased gut permeability, altered expression of tight junction molecules, activation of innate immune molecules, and an increase in activated adaptative immune cells. All those changes characterize a "leaky gut," the terminology used to collectively describe those gut alterations (De Kort et al., 2011). Together, these conditions can perturb gut homeostasis and increase the probability of developing an inflammatory disease such as Crohn's disease.

Currently, the best diet-based strategy for treating these conditions is a shift from a Western-style diet to a balanced, plant-based diet. However, the implementation of such changes takes time and requires a very high level of nutritional education. Because of this, the success rates of weight loss maintenance due to lifestyle changes are typically low (Lewis and Abreu, 2017). In response, alternative treatments are being developed that aim to ameliorate the negative gut effects. One group of treatments that has demonstrated promise are probiotics, which in certain cases have been shown to decrease both gut permeability and glycemia (Barengolts, 2016; Xu et al., 2019).

Faecalibacterium prausnitzii (F. prau) is a probiotic isolated from the human microbiota, where it is a dominant species in healthy adults. Its decline is associated with the development of chronic inflammation, as observed in cases of obesity. Multiple studies have demonstrated the anti-inflammatory properties of this bacterium (Feng et al., 2014; Andoh et al., 2016; Ganesan et al., 2018; Wrzosek et al., 2018), which are thought to be associated with its ability to produce butyrate. Butyrate activates the G-protein receptor (GPR) and thus facilitates downstream control of gut alterations during obesity and diabetes (Brown et al., 2003). Therefore, the goal of this review is to discuss the current state of knowledge regarding F. prau, particularly with respect to its potential as probiotic derived from human gut for use in alleviating the gut inflammation developed during obesity and hyperglycemia.

\section{OBESITY AND HYPERGLYCEMIA- ASSOCIATED GUT ALTERATIONS}

Obesity is a highly complex, multifaceted disease associated with numerous metabolic dysfunctions. These include type 2 diabetes mellitus (T2DM), dyslipidemia, cardiovascular dysfunction, and chronic inflammatory diseases (Winer et al., 2016). These conditions are linked with changes in adipose tissue (AT), a complex endocrine organ that plays an important role in energy homeostasis due to its rapid and dynamic responses to changes in nutrient availability (Sun et al., 2011). Adipose tissue is composed of adipocytes, macrophages, lymphocytes, fibroblasts, cell progenitors, and endothelial cells, and is responsible for the secretion of molecules such as leptin, adiponectin, cytokines, and the vascular regulators angiotensin II and plasminogen activator inhibitor (PAI-1) (Andersen et al., 2016).

In conditions of obesity, AT can become severely dysfunctional, with changes ranging from an increase in size to impaired function and atypical distribution in the body. This results in a suite of physiological alterations, including modifications to the extracellular matrix, vascularization, levels of oxidative stress, the profile of secreted adipokines, and the inflammatory state of infiltrated immune cells (Jo et al., 2009). Due to the increase in free fatty acids (FFA), signaling pathways such as IKK $\beta$ and NF- $\kappa \mathrm{B}$ are activated, along with those linked with Toll-like receptors (TLRs) (Crawford et al., 2009; Baker et al., 2011), which influence the inflammatory state. Obese patients are typically characterized by an increase in LPS that is accompanied by higher levels of TLR4 and CD14 expression, which all contribute to the proliferation of pro-inflammatory mechanisms (Winer et al., 2016).

The activation of TLRs is important in obesity, especially TLR4 (Winer et al., 2016), as these signaling pathways regulate the phosphorylation of proteins and lead to an increase in the production of molecules such as TNF- $\alpha$, IL-6, leptin, resistin, and chemokines like type 2 CC chemokine receptor (CCR2), which is related to monocyte migration. TNF- $\alpha$ promotes the activation of the NF-kB pathway, stimulation of the cell death signaling pathway, inhibition of the expression of the glucose transporter GLUT-4, and an increase in FFA levels and consequent reduction in insulin sensitivity (Gomez-Hernandez et al., 2016).

Compared to their lean counterparts, mice that are fed a highfat diet present a higher number of TCD $4^{+}$and $\mathrm{TCD} 8^{+}$cells and higher levels of IFN- $\gamma$ and TNF- $\alpha$, mainly in adipose tissue (Lumeng et al., 2007). The infiltration of $\mathrm{TCD}^{+}$cells in adipose tissue is followed by the accumulation of CX3CR $1^{\text {int }}$ macrophages, which migrate towards AT in response to greater amounts of FFAs, glucose, and apoptosis, thus increasing inflammation (Rocha et al., 2014). Adipose tissue macrophages (ATMs) infiltrate adipose tissue in a CCR2-dependent manner and inhibit the insulin signal in insulin-sensitive tissues, including liver, adipose tissue, and muscle (Hotamisligil, 2017; Kawano et al., 2016). Taken together, these conditions contribute to the production of pro-inflammatory cytokines and the release of monocyte 1 and 3 chemotactic protein (MCP-1 and MCP-3), which creates a cycle of continuous cell recruitment and constant inflammation in AT (Nishimura et al., 2009).

The inflammatory state in visceral adipose tissue, along with an excess of metabolites in the circulation, is a major driver of obesity-related insulin resistance (IR). IR is characterized by impaired phosphorylation of the insulin receptor in cells that depend on insulin. This results in increased serine phosphorylation of insulin receptor substrate 1 and 2 (IRS-1 and IRS-2) and activation of the SOCS (suppressor of cytokine signaling) protein, which reduces the insulin receptor's ability to transmit signals downstream in the insulin pathway and to capture glucose in cells (Biddinger and Kahn, 2006).

When the full extent of metabolic dysfunction is considered, the physiological impact of obesity is wide-ranging, with numerous effects on the architecture and functionality of primary and secondary immune system organs, including bone marrow, thymus, and lymph nodes (Yang et al., 2009). The accumulation of lipids reduces hematopoiesis in bone marrow and thymopoiesis in the thymus, which is added to a restricted diversity of $\mathrm{T}$ cell receptor repertoires in the thymus 
(Yang et al., 2009). In the peripheral immune response, there is a reduction in the migration of antigen-presenting cells to peripheral lymph nodes and a consequent reduction in the differentiation of naïve $\mathrm{T}$ cells into effective cells. These systemic effects can also translate into disturbances to the homeostasis of gut mucosa, which together with the mucosalassociated lymphoid tissue harbor the majority of immune cells found in the body.

The intestinal mucosa is the largest surface of the human body that is in contact with the external environment (Rezende and Weiner, 2017). The intestinal immune system is thus faced with an immense challenge: tolerating the vast amount of antigens that originate from the diet and the commensal microbiota while, at the same time, protecting against intestinal pathogens and toxins (Faria et al., 2017). Alterations in the intestinal barrier and dysbiosis in the gut of obese individuals may not only favor the development of diseases, but may also compromise immune tolerance to dietary antigens and the microbiota (Spiekermann and Walker, 2001; Faria and Weiner, 2005).

In the literature, there is an abundance of evidence on the many ways obesity and insulin resistance can affect immune responses and gut physiology. Obesity enhances jejunal inflammation and increases the density of macrophage populations, $\mathrm{CD} 3+\mathrm{T}$ cells, intraepithelial lymphocytes and mature dendritic cells. It also increases the expression of proinflammatory cytokines (IFN $\gamma$, IL1 $\beta$, TNFa), chemokines, and co-stimulatory factors in cells from the lamina propria and epithelial compartment. The increase in T-cell populations in the intestinal mucosa of obese subjects has also been associated with an impaired insulin response compared with lean subjects (Monteiro-Sepulveda et al., 2015). In humans with obesity and insulin resistance, there is a stronger inflammatory profile in the duodenum compared to non-obese patients, with more inflammatory cytokines and M1 macrophages (Ho-Plagaro et al., 2019). In addition, obesity and excess weight have been associated with increased gut permeability-demonstrated by increased serum concentrations of zonulin-along with microbiota modifications (Mörkl et al., 2018). Increased gut permeability was also reported in individuals with high levels of fasting glucose, as well as higher levels of pro-oxidative markers in the blood compared with healthier subjects (Carnevale et al., 2017). Furthermore, intestinal permeability was found to be sharply increased by a high-fat diet (HFD), probably mediated by a reduction in the expression of $\mathrm{ZO}-1$ and occludin, which then favors the translocation of LPS through the intestinal wall (Cani et al., 2008b).

Hyperglycemia associated with HFD increases levels of IFN$\gamma$ - and IL-17-producing inflammatory cells in the intestine and intestinal permeability, and decreases the abundance of regulatory cells (Luck et al., 2015). Even in the absence of excess weight, hyperglycemia has been associated with increased permeability and alterations in the mucosal immune system. For example, in $d b / d b$ mice with controlled food intake or in mice treated with streptozotocin, high levels of blood glucose were associated with high intestinal permeability and increased expression of PRP (pathogen recognition patterns) in lymphoid organs (Thaiss et al., 2018). In other words, hyperglycemia that is secondary to obesity, IR, or even T1D can further compromise intestinal health. Alterations in glucose metabolism can also decrease oral tolerance of antigens from the diet (Miranda et al., 2019) and increase the severity of food allergies (data not published) in mice.

One of the main consequences of the development of inflammatory alterations and the breakdown of gut epithelial integrity is the leakage of bacterial products, including endotoxins such as LPS, across the intestinal epithelium. This then results in dysfunction of immune organs, inadequate distribution of leukocyte populations, and changes in lymphocyte activity, which can all affect the immune response against pathogens, regulation of the immune response to dietary antigens, and the intestinal microbiota (Figure 1).

\section{CHANGES IN GUT MICROBIOTA RELATED TO OBESITY AND HYPERGLYCEMIA}

Composition of the microbiota is modulated by the availability of dietary nutrients that provide a wide variety of essential metabolites for the maintenance of intestinal architecture and integrity, while simultaneously acting on the modulation of immunity. In healthy conditions, Bacteroidetes and Firmicutes are the most abundant phyla of the microbial gut community. Although the structure of these communities can vary, some general patterns have been noted. For example, studies have determined that some of the most abundant intestinal bacterial species in healthy individuals tend to include members of the Dorea/Eubacterium/Ruminococcus groups as well as Bifidobacteria, Proteobacteria, and streptococci/ lactobacilli (Eckburg et al., 2005; Qin et al., 2010).

Diet is the key determinant of microbiota composition; it modulates the abundance of various species and, consequently, their individual or collective functions. In humanized gnotobiotic mice, a shift from a low-fat, plant polysaccharide-rich diet to a high-fat and high-sugar diet had detectable effects on microbial community structure and metabolic pathways after only a single day. More specifically, the increase in body fat percentage in mice fed a high-fat diet was positively associated with the abundance of species in the genera Lactococcus and Allobaculum but was negatively associated with Akkermansia (Kolodziejczyk et al., 2019).

The alterations in the microbiota linked with HFD-induced obesity also have effects on gut permeability to bacteria and bacterial products. The Burcelin group $(2008,2011)$ described that after 1 week of HFD consumption, changes could be seen in the intestinal mucosa and microbiota such as co-localization of bacteria with dendritic cells (DCs) both in the mucosa and in the mesenteric lymph nodes. After 4 weeks of HFD consumption, clear increases in intestinal permeability have been noted, with a concomitant decrease in zonulin expression in the intestine. These changes were also dependent on changes in the microbiota (Cani et al., 2008b; Amar et al., 2011). These alterations in the intestine permit increased bacterial translocation, which involves intestinal phagocytes and requires the recognition of pathogen-associated molecular 


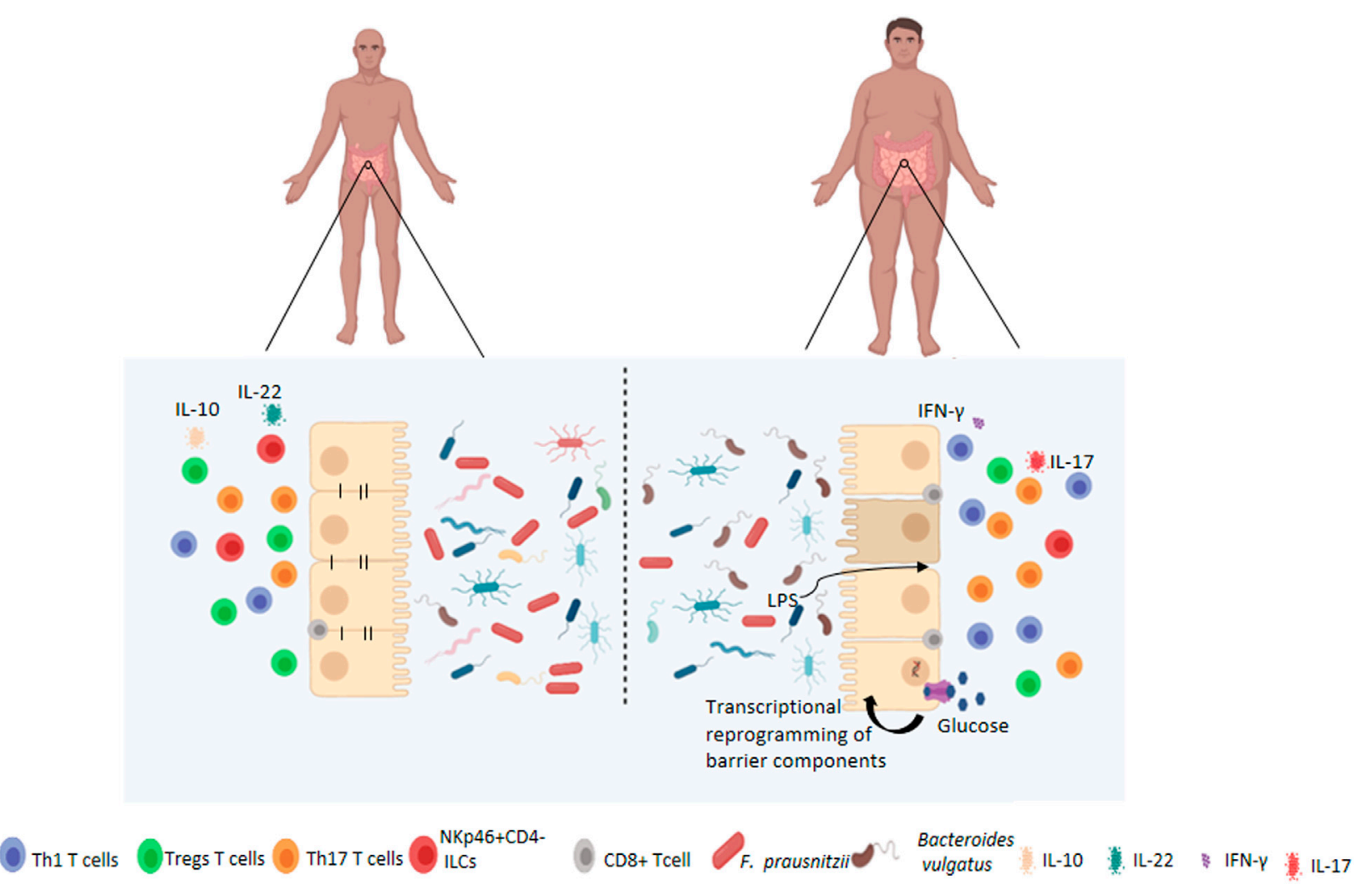

FIGURE 1 | Changes to the intestinal barrier functions associated with obesity. Under normal circumstances, the gut microbiota is highly diversified which contributes to the maintenance of intestinal permeability, tolerance to dietary antigens and immunoregulation. Obese patients have dysbiosis of the gut microbiota, characterised by decreased diversity, and altered barrier functions such as increased permeability and activation of pro-inflammatory pathways by immune cells at local and systemic levels. Obesity-associated hyperglycemia may also drive barrier permeability through transcriptional reprogramming resulting in decreased expression of adherens and tight juction proteins.

patterns (PAMPs) by standard recognition receptors (PRRs), such as TLRs and nod-like receptors, to activate an innate immune response (Kim et al., 2012; Luck et al., 2015). Under pathological conditions. However, this molecular signaling process can favor further infiltration and cell accumulation in adipose tissue, triggering local inflammation.

The relationship between dysbiosis and gut permeability also plays a role in the development of insulin resistance. Specifically, alterations in the abundance of intestinal bacteria caused by chronically elevated glucose levels and obesity are known to result in dysfunction in the intestinal barrier (Guerville et al., 2017). As a result, high concentrations of gram-negative bacterial cell-wall products, such as LPS, can cross the intestinal barrier to reach other organs and tissues, and induce chronic inflammation (Cani et al., 2009). This can initiate a series of inflammatory mechanisms, setting in motion one of the main processes that leads to insulin resistance and ultimately T2D (Cani et al., 2007; Ganesan et al., 2018). This can be further exacerbated by the fact that the production of pro-inflammatory cytokines interferes with insulin secretion and the expression of insulin mRNA in human beta islet cells. Changes in the composition of the microbiota can thus play a multi-faceted role in intestinal barrier dysfunction and, consequently, in metabolic disorders.

Certain groups of bacteria have been linked with various conditions in the gut. As mentioned earlier, an increased ratio of Firmicutes to Bacteroidetes in obese individuals has been related to inflammatory diseases (Turnbaugh et al., 2006), while conversely, the ratio of Bacteroides to Prevotella is lower in obese subjects than their lean counterparts. F. prau has been negatively associated with insulin resistance (Furet et al., 2010), while potential proinflammatory bacteria such as Ruminococcus gnavus or Bacteroides may dominate the microbiota of obese patients. In general, a reduction of butyrate-producing bacteria in obese subjects has been associated with an increase in mucus degradation (Cotillard et al., 2013). Obese women with high serum levels of zonulin, which is correlated with higher gut permeability, showed decreased abundance of Ruminococcaceae and Faecalibacteri both could weaken the gut barrier and lead to systemic inflammatory responses (Mörkl et al., 2018).

A study in mice reported that, after 1 week of HFD, and the subsequent early onset of diabetes, gram-negative bacteria start to adhere in the DCs of the gut mucosa; this increased bacterial 
translocation and triggered inflammation (Amar et al., 2011). Interestingly, mice that are deficient in TLR5, which recognizes bacterial flagellin, develop obesity and features of metabolic syndrome even in the absence of a high-fat diet, solely as a function of microbiota changes (Vijay-Kumar et al., 2010). Finally, some bacterial species have been identified as particular promoters of insulin resistance, for example, Prevotella copri and Bacteroides vulgatus, which were noted to be the main species driving the association between the biosynthesis of branched-chain amino acids and insulin resistance in humans (Pedersen et al., 2016).

One important function of the microbiota is the metabolism of polysaccharides, through which these bacteria produce a wide variety of metabolites, such as short-chain fatty acids (SCFAs), that are essential for the microbial population and for the maintenance of intestinal homeostasis (Sun et al., 2017). The SCFAs acetate, butyrate, and propionate are the main products of fermentation in the intestine, and are of particular interest due to their ability to activate local G-protein-coupled receptors (GPCR) in epithelial cells, especially GPR41 and GPR43 (Miyamoto et al., 2016). This process has feedback effects on the physiology of the intestinal microbiome and mediates chronic inflammation, affecting both glucose homeostasis and insulin sensitivity (Ang and Ding, 2016; Lambertz et al., 2017). Butyrate-producing bacteria such as $F$. prau can reduce bacterial translocation and stimulate mucin secretion, which acts to maintain the integrity of the intestine (Ganesan et al., 2018).

Interactions between the intestinal microbiota and host cells require finely tuned control by the immune system, as specialized cells are needed to recognize bacterial fragments and induce inflammation when appropriate. A significant consequence of the microbial changes associated with obesity is activation of the innate immune system, which often results in chronic inflammation (Cani et al., 2007; Cani et al.,2008a). In animal models, increased levels of LPS in the bloodstream can directly damage pancreatic $\beta$ cells and increase insulitis by triggering the innate immune response; this is a crucial factor in the pathogenesis of insulin resistance (Ganesan et al., 2018). Similarly, Amar et al. (2011) reported that excess LPS fragments in the blood of diabetic mice induced adipose tissue inflammation.

Interestingly, when HFD experiments are performed in animals lacking the microbial pattern recognition receptors Nod1 or CD14, diet no longer has strong effects on bacterial permeability and translocation (Amar et al., 2011). Likewise, TLR4-knockout mice have a completely different microbiota and display elevated blood LPS levels and diabetes development compared to WT controls. These alterations in gut morphology and microbiota composition are also correlated with lower levels of circulating SCFAs, which suggests an interaction between intestinal functions, microbiota composition, and the development of diabetes (Simon et al., 2020).

Taken together, the changes to the structure and function of the gut microbiota that occur in cases of obesity and hyperglycemia have the potential to severely exacerbate mucosal inflammation and gut permeability. For this reason, the use of bacterial probiotics-especially those known to produce high levels of SCFAs-could represent an interesting approach to treat the damage associated with intestinal metabolic syndrome.

\section{PROBIOTICS AS AN ALTERNATIVE TREATMENT FOR OBESITY AND HYPERGLYCEMIA}

Modulation of the microbiota has become a regular and effective approach in the treatment and prevention of mucositis, colorectal cancer, neurological diseases, and several other disorders (Sanders et al., 2019). There are several ways to modify the microbiota, including diet alteration; the administration of probiotics, prebiotics, and postbiotics; and fecal microbiota transplantation. The underlying goal of all of these methods is the same: to modify the bacterial composition in the gut in order to provide benefits to the host. As the links between obesity, hyperglycemia, and alterations in the microbiota have become clearer, studies have attempted to shed light on the exact mechanisms by which diet is able to affect the microbiome. It is possible to envision that microbiota modulation could serve as a non-invasive means of treating metabolic conditions, especially obesity, and thus represent an attractive alternative to common approaches such as bariatric surgery.

As mentioned above, obesity-related dysbiosis induces a series of events, mainly in the intestine, that result in chronic inflammation and disruptions to intestinal homeostasis. However, until the early 2000s, there was no discussion of the possibility that the microbiota itself could contribute to weight gain (Backhed et al., 2004). The Gordon group has conducted research on this topic, and their pioneering studies proposed an interesting hypothesis-that the microbiome from obese subjects has an increased capacity to harvest energy from the diet. First, it was reported that control mice colonized with an "obese" microbiota present higher body fat compared to mice colonized with "lean" microbiota (Turnbaugh et al., 2006). Further studies with gnotobiotic germ-free C57BL/6 mice revealed that a Western diet induced a drop in microbiota diversity, with an increase in abundance of a single class of Firmicutes. In addition, when the microbiota from conventionally raised obese mice were transplanted into wildtype mice, they showed a greater increase in body fat (Turnbaugh et al., 2008). Finally, a fascinating study with adult monozygotic and dizygotic twins showed that, although twins shared a core microbiota, obese siblings had less diversity in their microbial assemblages, lower proportions of Bacteroidetes, and a higher proportion of Actinobacteria compared to their leaner twins (Turnbaugh et al., 2009). These interesting findings have led to the generation of several hypotheses, with one of the most intriguing being that microbiota modulation by itself might be an effective treatment for obesity.

The relationship between gut microbiota and energy balance (and thus the development of larger adipose tissue) is complex because it involves many factors, such as diet, gender, and culture, among others. However, recent studies have pointed to the gut 
microbiome as a key element in the regulation of food absorption; there is evidence that the gut microbiota can affect hormone secretion directly in the brain, in areas that are responsible for controlling appetite, fat storage, and energy expenditure (Cerdó et al., 2019). Although the science is far from settled on the topic, based on these initial results, some attempts have been made to treat obesity by modifying the host microbiota.

To date, probiotics and prebiotics have been used for the treatment of obesity in both experimental models and clinical trials. Probiotics are live microorganisms that, when ingested, can confer benefits on the host, while prebiotics are molecules or components (such as food fiber) that are capable of modifying the composition of the intestinal bacterial assemblage or stimulating the activity of one or a limited number of bacterial species in a positive way (Gibson et al., 2017). In most cases, one of the noted benefits of both prebiotics and probiotics is increased levels of SCFAs, which have regulatory effects and are important for intestinal integrity. Much research has focused on the positive effects of Lactobacillus and Bifidobacterium in obesity; both bacteria have been reported to contribute to weight loss and a reduction in insulin resistance. For instance, several studies involving different mouse or rat models of obesity have shown positive results from treatment with certain strains of $L$. plantarum (DSM 15313, Strain No. 14, and TL8), such as less weight gain, reduced adiposity, and higher insulin sensitivity (Axling et al., 2012; Ben Salah et al., 2013; Okubo et al., 2013). A recent publication reported that L. plantarum LMT1-48 significantly reduced weight in HFD-fed mice and its extract was capable of inhibiting the differentiation of adipocytes through downregulation of genes such as PPAR- $\gamma$ (Choi et al., 2020). Notably, synergistic effects were observed from a probiotic combination of L. plantarum KY1032 and L. curvatus HY7601; HFD-fed mice treated with this mixture showed reduced body weight gain and fat accumulation, lower levels of insulin and cholesterol, and fewer biomarkers for inflammation (Park et al., 2013; Yoo et al., 2013). One exception was reported with HFD-fed mice treated with L. plantarum DSM 15313, which showed an increase in body weight despite lower levels of glucose in plasma (Andersson et al., 2010). Another study, with L. plantarum NCIMB8826, found no effect on body weight (Martinic et al., 2018). Overall, numerous strains-including L. paracasei CNCM I-4034, L. casei IMVB-7280, L. paracasei HII01, L. casei IBS041, L. rhamnosus CGMCC1.3, L. rhamnosus PB01 (DSM 14870), and L. rhamnosus LA68, among others-have demonstrated probiotic potential in studies of obesity, inducing positive effects such as reduced weight gain, less adiposity with less white adipose tissue, and reduced cholesterol levels, as reviewed by Ejathed et al. (Ejtahed et al., 2019).

Avolio and colleagues et al showed that HFD-fed hamsters treated with a probiotic mix of six species (S. thermophilus, $L$. bulgaricus, L. lactis, L. plantarum, B. lactis, and L. reuteri) presented a reduction in body weight and reduced levels of inflammatory factors in the blood compared to control HFD animals (Avolio et al., 2019). A study of leptin-deficient mice (ob/ ob mice) showed that administration of the plant-derived lactic acid bacterium Pediococcus pentosaceus was sufficient to reduce adipocyte size and liver triglyceride content (Zhao et al., 2012).
Similarly, HFD-fed mice treated with $L$. plantarum demonstrated reduced adipose tissue and triglyceride levels; the treatment also reduced the Firmicutes/Bacteroidetes ratio and improved the gut microbiota composition (Joung et al., 2021). Finally, although most effort to date has focused on bacteria, the most-studied yeast probiotic, Saccharomyces boulardii, has been reported to reduce hepatic steatosis and hepatic inflammation in $\mathrm{db} / \mathrm{db}$ mice (Everard et al., 2014).

Positive results are also being reported from obese mice treated with various types of prebiotics. For example, the use of oligofructose as a prebiotic in HFD-fed obese mice resulted in higher numbers of intestinal Bifidobacteria and Lactobacillus in treated mice; this correlated with higher zonulin expression, leading to less intestinal permeability and less hepatic inflammation (Cani et al.,2008b). The addition of oligofructose in the diet was also found to promote satiety in HFD-fed mice, contributing to a reduction in both weight and adipose tissue deposits (Cani et al., 2005; Régnier et al., 2021). Another study showed that short-chain fructose-oligosaccharides induced a significant increase in the abundance of Bifidobacteria in obese mice, and these animals gained less weight than control HFD mice (Respondek et al., 2013). In general, the use of prebiotics as a treatment for obesity seems quite promising; this is especially true for approaches using food fiber, which seems to increase the production of satiety hormones, thus helping to control weight and increasing sensitivity to insulin (Parnell et al., 2012).

In humans, several clinical trials of probiotics and prebiotics as a treatment for obesity have reported positive results. For example, a randomized, double-blind study conducted by Osterberg and collaborators demonstrated that administration of a probiotic containing eight strains of bacteria (Lactobacillus, Bifidobacterium, and Streptococcus) attenuated increases in body mass index (BMI) and adipose tissue in subjects on a high-fat diet (Boutagy et al., 2015). Similarly, other studies of interventions with Lactobacillus spp. and Bifidobacterium spp. have reported positive results in obese and overweight subjects, such as less body weight and less fat storage (Minami et al., 2015; Madjd et al., 2017). Two recent systematic reviews concluded that some probiotic strains are effective in reducing BMI and hip circumference (Shirvani-Rad et al., 2021; Tomé-Castro et al., 2021).

Despite the promising results thus far, there is still much room for new approaches and improvements, especially those aimed at elucidating the mechanisms by which various probiotics and prebiotics induce reductions in weight and inflammation. In this context, the probiotic derived from human gut F. prau may prove particularly useful.

\section{F. PRAU AS A NEW TREATMENT TO IMPROVE GUT HOMEOSTASIS DURING OBESITY AND IR}

F. prau is a Gram-positive bacterium belonging to the Ruminococcaceae family, in Clostridium cluster IV. It is one of the most abundant species found in the gut, representing between 1 and $6 \%$ of the total fecal microbiota (Hold et al., 2003). Multiple 
studies have described its anti-inflammatory properties and its role in tissue damage repair and protection against colitis(Sokol et al., 2008; Martín et al., 2014; Rossi et al., 2015), which appear to be related, at least in part, to its ability to produce butyrate(Zhou et al., 2018). F. prau is characterized by great intra-species diversity; indeed, it has been suggested that the genomic disparities are great enough to warrant separating the group into at least two different species: $F$. prau sensu stricto and $F$. moorei sp. nov (Fitzgerald et al., 2018).

Patterns of abundance of $F$. prau and its different phylogroups have been associated with various pathologies of the gut. For example, IBD patients were found to host reduced phylotype richness of $F$. prau compared to a healthy group. Specifically, total levels of $F$. prau phylogroup I were reduced, and this pattern could be used to accurately differentiate IBD and colorectal cancer patients from healthy subjects; instead, phylogroup II was specifically reduced in Crohn's disease (CD) patients. Interestingly, $F$. prau prevalence was found to be reduced locally in either the ileum, colon, or rectum depending on the form of the patient's CD (Lopez-Siles et al., 2016). Taken together, this demonstrates the potential applications of $F$. prau phylotypes as biomarkers for the diagnosis and prognosis of patients.

As discussed earlier, diversity of the microbiota is lower in obese patients, and F. prau has been implicated in these changes in community composition. For example, analysis of a Chinese cohort revealed a reduced abundance of Bacteroides, Akkermansia (another butyrate-producing bacterium), and $F$. prau in T2D patients. Evidence of this shift was even detectable in pre-diabetic obese patients, reflecting the link between glucose intolerance and microbiota composition (Zhang et al., 2013). These results-especially with respect to F. prau-were corroborated in a later Iranian study, which also reported a negative correlation between $F$. prau count and BMI (Navab-Moghadam et al., 2017). In another study, though, F. prau was found to be enriched in patients suffering from T2D after weight loss (Hippe et al., 2016). Thus, although the exact mechanisms have yet to be determined, the current state of research supports a link between BMI, blood glucose levels, and the abundance of $F$. prau.

Another interesting finding with respect to $F$. prau was the observation of differences in gut composition on the basis of gender, especially for this bacterium and streptococci. In a population of obese Chinese patients, a positive correlation was found between $F$. prau abundance and fasting glucose levels in men but not in women (Aguirre de Cárcer et al., 2011).

Rapid improvements (on the scale of a few days) have been observed in the community structure of gut microbiota as a result of nutritional interventions. Diets rich in non-digestible carbohydrates, such as inulin-type fructans, fructooligosaccharides, polydextrose, soluble corn fiber, and raffinose, have been observed to increase the abundance of $F$. prau (Verhoog et al., 2019). Supplements can also be effective; for example, kiwifruit-based supplementation was noted to increase $F$. prau abundance in the gut as well as stool frequency in humans (Rush et al., 2002; Blatchford et al., 2017).
Alternatively, supplementation with $F$. prau itself could have potential in the treatment of obesity and its associated disorders. HFD-fed mice that were treated twice a week with $F$. prau displayed decreased hepatic inflammation, with fewer lipids accumulated in the liver, as well as a reduction in cell infiltration of adipose tissue and adipocyte size compared to controls (Munukka et al., 2017).

Finally, many studies have examined the mechanisms by which $F$. prau exerts its beneficial impacts on the intestinal health of obese individuals (Figure 2). It appears that these effects may not only be a function of the abundance of this bacterium in the intestine, but also of the quantity and type of metabolites it produces, such as butyrate. Indeed, some of these metabolites have already demonstrated promise for the treatment of obesity and T2D (Ganesan et al., 2018; Verhoog et al., 2019).

\section{F. PRAU ACTION THROUGH BUTYRATE PRODUCTION}

F. prau is one of the most abundant butyrate-producing bacteria in human feces (Hold et al., 2003), and this SCFA is currently the subject of intense research focused on its positive health effects. For example, butyrate can prevent HFD-induced insulin insensitivity through epigenetic regulation that increases mitochondrial beta-oxidation, thus improving glucose sensitivity and adiposity (Fernandes et al., 2014).

The butyrate-containing supernatant of $F$. prau has been found to regulate Th17/Treg differentiation through inhibition of the IL-6 and STAT3/IL-17 proinflammatory pathway, specifically by targeting histone deacetylase 1 (HDAC1) (Rivière et al., 2016; Zhou et al., 2018). Although this mechanism was demonstrated in a colitis model, this proinflammatory pathway is also involved in obesity (Hippe et al., 2016). These results are consistent with the observation that HFD-mice treated with F. prau have improved hepatic health and reduced inflammation in adipose tissue (Munukka et al., 2017).

The anti-inflammatory properties of butyrate, and by extension $F$. prau, have long been known to be beneficial to IBD patients(JM et al., 1989; W et al., 1992; Scheppach, 1996). A recent clinical study found that administration of encapsulated sodium butyrate altered patients' gut microbiota by increasing the abundance of SCFA-producing bacteria in ulcerative colitis patients and butyrate-producing bacteria in Crohn's disease patients, with the former group reporting an increased quality of life (Facchin et al., 2020). Sodium butyrate supplementation also had positive effects on HFD-fed mice by altering the composition of the gut microbiota, lowering serum LPS concentration, and reducing $\mathrm{HFD}$-induced inflammation (Zhou et al., 2017).

The A2-165 strain of $F$. prau has been found to induce a distinct cytokine response, with high IL-10 secretion compared to other F. prau strains tested (Rossi et al., 2016). This may be the result of higher butyrate production, which is known to induce IL-10 responses in Th1 cells (Sun et al., 2018). Indeed, a recent study demonstrated that this strain's anti-inflammatory 

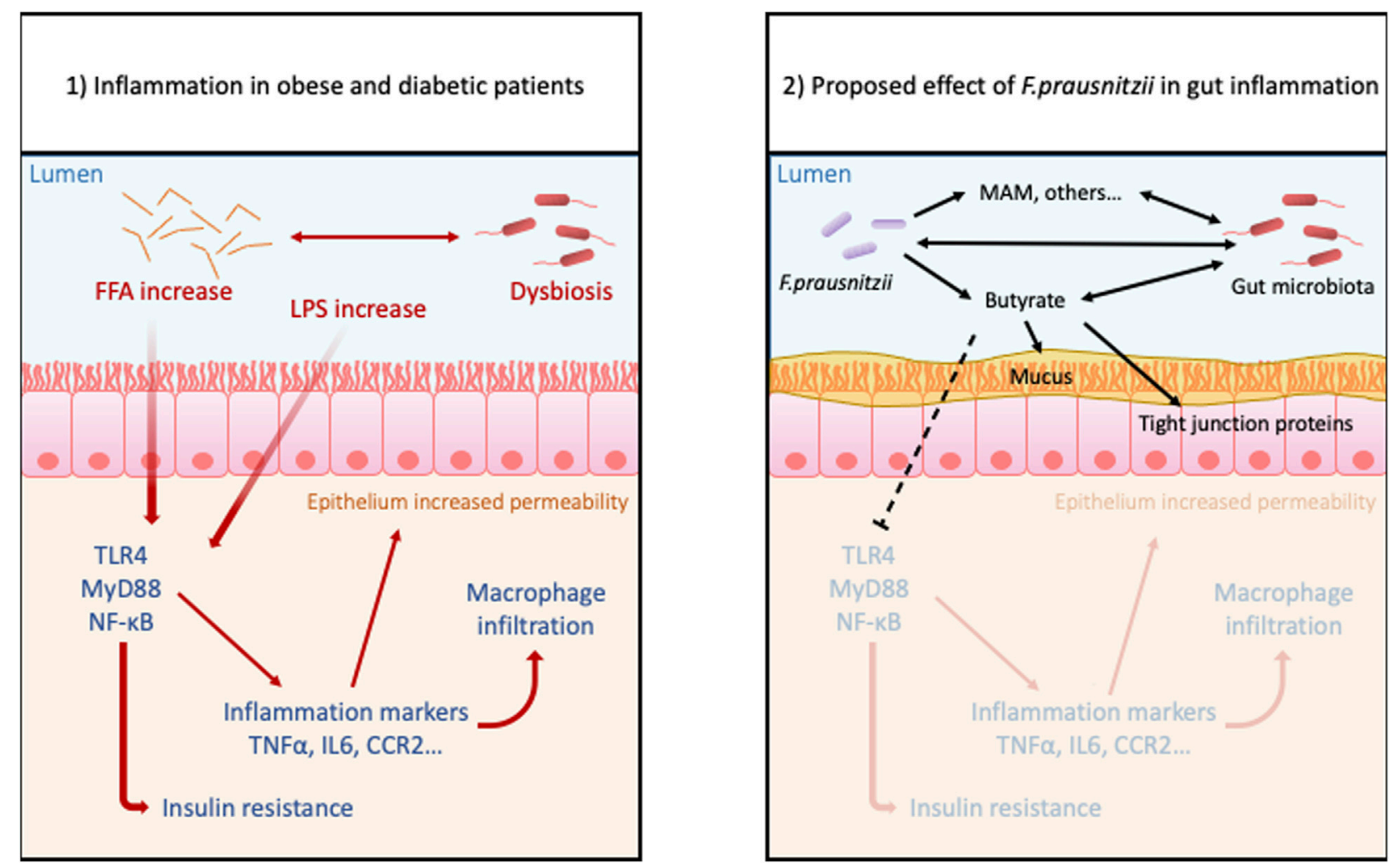

FIGURE 2 | Proposed benefits effects of Faecalibacterium prausnitzii in gut inflammation due to obesity: Obesity and hyperglycemia cause dysbiosis, increased level of FFA and LPS, and also disrupts intestinal permeability. These conditions trigger inflammatory pathways in the lamina propria allowing more permeability to antigens and bacteria. This leads to a leaky gut and susceptibility to inflammatory disorders. The treatment with probiotic F. prau and its products can increase the butyrate concentration, stabilizes the microbiota and mucous layer and decreases the activation of inflammatory pathway.

properties in primary human colonic mucosal barrier cells are primarily due to the downregulation of TLR3 and TLR4 by butyrate (Zhang et al., 2021). It thus seems likely that differences in the cytokine profile induced by strain A2-165 compared to other strains of $F$. prau can be at least partially explained by higher butyrate production.

However, gaps remain in our understanding of F. prau's butyrate metabolism and its effects on patients' health, with some studies reporting inconsistent results. For example, an early study in southern India found significantly higher abundances of F. prau in obese children compared to nonobese children (Balamurugan et al., 2010). Similarly, Pinto et al. (2017) found increased levels of F. prau in T1D patients. They showed that the high abundance of the gluconeogenic enzyme phosphenolpyruvate carboxykinase in the gut proteome of their T1D cohort could be attributed to two strains of $F$. prau and hypothesized that this was due to low levels of glycolytic sources in the diets of T1D patients and a lack of acetate-necessary for butyrate production-from other bacterial sources such as Bifidobacterium spp. (Pinto et al., 2017). This hypothesis was supported by the finding that coculture with strains of Bifidobacterium improved growth, gut colonization, and butyrate production of F. prau. Administration of the co-culture supernatant to mice decreased DSS-induced inflammation, providing further evidence that the beneficial properties of $F$. prau are dependent on its surrounding environment and interactions with other species (Ganesan et al., 2018; Kim et al., 2020).

Intriguingly, a 2016 study reported that samples from lean patients contained the highest count of $F$. prau genes compared to obese and T2D patients, but the lowest content of the $F$. prau-associated butyryl-CoA:acetate CoA-transferase (BUT) gene. Instead, T2D patients demonstrated the highest BUT content. This was interpreted as evidence that different phylotypes of $F$. prau produce different levels of butyrate in vivo and that their abundance differs from healthy to unhealthy patients (Hippe et al., 2016). It seems paradoxical that higher butyrate production by $F$. prau would be associated with obesity and T2D. The authors hypothesized that, while certain levels of butyrate production can be protective in obese patients, greatly increased production can lead to inflammation in the gut and the development of T2D. The dose-dependent effect of butyrate on epithelium permeability has long been supported by work using transepithelial electrical resistance measurements; experiments on Caco-2 cells showed protective effects at $2 \mathrm{mM}$ and detrimental effects at $8 \mathrm{mM}$ of butyrate (Peng et al., 2007). Similarly, a Belgian study on primary cell monolayers from ulcerative colitis patients found that although butyrate had 
protective effects in control non-inflamed tissue, it actually worsened inflammation when administered together with TNF-alpha and IFN-gamma, resulting in a dramatic increase in IL-8 production (Vancamelbeke et al., 2019).

F. prau has been associated with the production of several other metabolites, including shikimic and salicylic acids, known for their antimicrobial activity, and $\alpha$-ketoglutaric acid, which is involved in ammonia recycling and cell proliferation and differentiation, and known to be depleted in patients with gut dysbiosis (Miquel et al., 2015). Investigation of these other metabolites has become all the more relevant given a report that the ability of 13 different strains to decrease IL-8 levels induced by TNF- $\alpha$ stimulation in HT-29 cells was not correlated either to growth ratio or butyrate production (Martín et al., 2017).

Quévrain et al. (2016) identified a family of peptides from $F$. prau that were all derived from the same protein, the microbial anti-inflammatory molecule (MAM). They demonstrated that the anti-inflammatory properties of MAM arose through inactivation of the NF- $\kappa$ B pathway. Later experiments in models of DNBSand DSS-induced colitis validated the effects of MAM on NF- $\kappa \mathrm{B}$ in vivo and demonstrated its ability to inhibit the Th1 and Th17 immune responses (Breyner et al., 2017).

A more recent investigation of MAM analyzed its effects in $\mathrm{db} /$ $\mathrm{db}$ mice, which do not express leptin receptors and which demonstrated a depressed abundance of F. prau in the gut. When these mice were supplemented with MAM produced by E. coli, this protein was found to interact with ZO-1 and other tight junction proteins. Furthermore, the transfection of MAM into a cell line was able to increase ZO-1 expression and restore epithelial barrier function (Xu et al., 2019). These results suggest that MAM could have potential as an alternative treatment for pathologies involving disturbances of the gut epithelium and gut permeability.

It has thus become evident that butyrate is far from the only metabolite implicated in the immunomodulatory properties of $F$. prau. The use of this bacterium as a preventive or complementary treatment for obesity- and T2D-related inflammation of the gut could be promising. However, we first need a better understanding of the optimal dosage of bacterial units and the effects of butyrate production on host health, as well as how $F$. prau and butyrate metabolism are affected by other gut bacteria.

\section{REFERENCES}

Aguirre de Cárcer, D., Cuív, P. O., Wang, T., Kang, S., Worthley, D., Whitehall, V., et al. (2011). Numerical Ecology Validates a Biogeographical Distribution and Gender-Based Effect on Mucosa-Associated Bacteria along the Human colon. ISME J. 5, 801-809. doi:10.1038/ismej.2010.177

Amar, J., Chabo, C., Waget, A., Klopp, P., Vachoux, C., Bermúdez-Humarán, L. G., et al. (2011). Intestinal Mucosal Adherence and Translocation of Commensal Bacteria at the Early Onset of Type 2 Diabetes: Molecular Mechanisms and Probiotic Treatment. EMBO Mol. Med. 3, 559-572. doi:10.1002/emmm.201100159

Andersen, C. J., Murphy, K. E., and Fernandez, M. L. (2016). Impact of Obesity and Metabolic Syndrome on Immunity. Adv. Nutr. 7, 66-75. doi:10.3945/ an.115.010207

Andersson, U., Bränning, C., Ahrné, S., Molin, G., Alenfall, J., önning, G., et al. (2010). Probiotics Lower Plasma Glucose in the High-Fat Fed C57BL/6J Mouse. Benef. Microbes 1, 189-196. doi:10.3920/BM2009.0036

\section{FINAL CONSIDERATION}

Obesity is a metabolic disease caused by several factors-genetic, environmental, hormonal, and behavioral-but mainly by excess energy intake. It predisposes patients to other diseases such as hypertension and type 2 diabetes, and it is also associated with disorders of intestinal homeostasis, such as increased permeability and dysbiosis. Non-surgical treatments for obesity include changes in lifestyle, diet, and medications, all of which tend to have low adherence by the patient and are rarely effective for weight control or even for shaping intestinal health. As a major factor associated with obesity and gut health, intestinal dysbiosis may be the key to the effectiveness of weight management and the control of its associated comorbidities. For this reason, several probiotics have been tested successfully for the control of obesity, and there is intense interest in the discovery of new potential treatments.

F. prau is well known as an abundant bacterium in the natural human microbiota whose abundance is reduced in obese individuals. In addition to being a high producer of butyrate, it has anti-inflammatory effects that contribute to intestinal homeostasis. Therefore, the use of $F$. prau or its derivative products may represent a good alternative for the treatment of intestinal disorders linked with obesity and its comorbidities. However, studies on dose, forms of administration, and mechanisms of action are still necessary in order to improve our understanding of the most appropriate use of this bacterium.

\section{AUTHOR CONTRIBUTIONS}

TM contributed to design of the study and wrote the article. EB-N wrote sections of the article and drew the figure. LT, $\mathrm{SB}$, and VM wrote sections in the first draft of the article. VA, PL and J-MC supervised and made critical reviews of the article. All authors approved the submitted version.

\section{FUNDING}

This work was supported by Coordenação de Aperfeiçoamento de Pessoal de Nível Superior (CAPES)-CAPES-COFECUB 934/19.

Andoh, A., Nishida, A., Takahashi, K., Inatomi, O., Imaeda, H., Bamba, S., et al. (2016). Comparison of the Gut Microbial Community between Obese and Lean Peoples Using 16S Gene Sequencing in a Japanese Population. J. Clin. Biochem. Nutr. 59, 65-70. doi:10.3164/jcbn.15-152

Ang, Z., and Ding, J. L. (2016). GPR41 and GPR43 in Obesity and Inflammation - Protective or Causative? Front. Immunol. 7, 28. doi:10.3389/fimmu.2016.00028

Arroyo-Johnson, C., and Mincey, K. D. (2016). Obesity Epidemiology Worldwide. Gastroenterol. Clin. North. Am. 45, 571-579. doi:10.1016/j.gtc.2016.07.012

Avolio, E., Fazzari, G., Zizza, M., De Lorenzo, A., Di Renzo, L., Alò, R., et al. (2019). Probiotics Modify Body Weight Together with Anxiety States via Proinflammatory Factors in HFD-Treated Syrian golden Hamster. Behav. Brain Res. 356, 390-399. doi:10.1016/j.bbr.2018.09.010

Axling, U., Olsson, C., Xu, J., Fernandez, C., Larsson, S., Ström, K., et al. (2012). Green tea Powder and Lactobacillus Plantarum Affect Gut Microbiota, Lipid Metabolism and Inflammation in High-Fat Fed C57BL/6J Mice. Nutr. Metab. (Lond) 9, 105-118. doi:10.1186/1743-7075-9-105 
Bäckhed, F., Ding, H., Wang, T., Hooper, L. V., Koh, G. Y., Nagy, A., et al. (2004). The Gut Microbiota as an Environmental Factor that Regulates Fat Storage. Proc. Natl. Acad. Sci. U S A. 101, 15718-15723. doi:10.1073/pnas.0407076101

Baker, R. G., Hayden, M. S., and Ghosh, S. (2011). NF-кB, Inflammation, and Metabolic Disease. Cell Metab 13, 11-22. doi:10.1016/j.cmet.2010.12.008.NF-

Balamurugan, R., George, G., Kabeerdoss, J., Hepsiba, J., Chandragunasekaran, A. M., and Ramakrishna, B. S. (2010). Quantitative Differences in Intestinal Faecalibacterium Prausnitzii in Obese Indian Children. Br. J. Nutr. 103, 335-338. doi:10.1017/S0007114509992182

Barengolts, E. (2016). Gut Microbiota, Prebiotics, Probiotics, and Synbiotics in Management of Obesity and Prediabetes: Review of Randomized Controlled Trials. Endocr. Pract. 22, 1224-1234. doi:10.4158/EP151157.RA

Ben Salah, R., Trabelsi, I., Hamden, K., Chouayekh, H., and Bejar, S. (2013). Lactobacillus Plantarum TN8 Exhibits Protective Effects on Lipid, Hepatic and Renal Profiles in Obese Rat. Anaerobe 23, 55-61. doi:10.1016/ j.anaerobe.2013.07.003

Biddinger, S. B., and Kahn, C. R. (2006). FROM MICE TO MEN: Insights into the Insulin Resistance Syndromes. Annu. Rev. Physiol. 68, 123-158. doi:10.1146/ annurev.physiol.68.040104.124723

Blatchford, P., Stoklosinski, H., Eady, S., Wallace, A., Butts, C., Gearry, R., et al. (2017). Consumption of Kiwifruit Capsules Increases Faecalibacterium Prausnitzii Abundance in Functionally Constipated Individuals: a Randomised Controlled Human Trial. J. Nutr. Sci. 6, e52. doi:10.1017/jns.2017.52

Boutagy, N. E., Neilson, A. P., Osterberg, K. L., Smithson, A. T., Englund, T. R., Davy, B. M., et al. (2015). Probiotic Supplementation and Trimethylamine-NOxide Production Following a High-Fat Diet. Obesity (Silver Spring) 23, 2357-2363. doi:10.1002/oby.21212

Breyner, N. M., Michon, C., de Sousa, C. S., Vilas Boas, P. B., Chain, F., Azevedo, V. A., et al. (2017). Microbial Anti-inflammatory Molecule (MAM) from Faecalibacterium Prausnitzii Shows a Protective Effect on DNBS and DSSInduced Colitis Model in Mice through Inhibition of NF-Kb Pathway. Front. Microbiol. 8, 114. doi:10.3389/fmicb.2017.00114

Brown, A. J., Goldsworthy, S. M., Barnes, A. A., Eilert, M. M., Tcheang, L., Daniels, D., et al. (2003). The Orphan G Protein-Coupled Receptors GPR41 and GPR43 Are Activated by Propionate and Other Short Chain Carboxylic Acids. J. Biol. Chem. 278, 11312-11319. doi:10.1074/jbc.M211609200

Cani, P. D., Amar, J., Iglesias, M. A., Poggi, M., Knauf, C., Bastelica, D., et al. (2007). Metabolic Endotoxemia Initiates Obesity and Insulin Resistance. Diabetes 56, 1761-1772. doi:10.2337/db06-1491

Cani, P. D., Bibiloni, R., Knauf, C., Waget, A., Neyrinck, A. M., Delzenne, N. M., et al. (2008b). Changes in Gut Microbiota Control Metabolic EndotoxemiaInduced Inflammation in High-Fat Diet-Induced Obesity and Diabetes in Mice. Diabetes 57, 1470-1481. doi:10.2337/db07-1403

Cani, P. D., Neyrinck, A. M., Maton, N., and Delzenne, N. M. (2005). Oligofructose Promotes Satiety in Rats Fed a High-Fat Diet: Involvement of Glucagon-like Peptide-1. Obes. Res. 13, 1000-1007. doi:10.1038/oby.2005.117

Cani, P. D., Possemiers, S., Van De Wiele, T., Guiot, Y., Everard, A., Rottier, O., et al. (2009). Changes in Gut Microbiota Control Inflammation in Obese Mice through a Mechanism Involving GLP-2-Driven Improvement of Gut Permeability. Gut 58, 1091-1103. doi:10.1136/gut.2008.165886

Cani, P. D., Bibiloni, R., Knauf, C., Waget, A., Neyrinck, A. M., Delzenne, N. M., et al. (2008a). Changes in Gut Microbiota Control Metabolic EndotoxemiaInduced Inflammation in High-Fat Diet-Induced Obesity and Diabetes in Mice. Diabetes 57, 1470-1481. doi:10.2337/db07-1403.Additional

Carnevale, R., Pastori, D., Nocella, C., Cammisotto, V., Baratta, F., Del Ben, M., et al. (2017). Low-grade Endotoxemia, Gut Permeability and Platelet Activation in Patients with Impaired Fasting Glucose. Nutr. Metab. Cardiovasc. Dis. 27, 890-895. doi:10.1016/j.numecd.2017.06.007

Cerdó, T., García-Santos, J., G. Bermúdez, M., and Campoy, C. (2019). The Role of Probiotics and Prebiotics in the Prevention and Treatment of Obesity. Nutrients 11, 635. doi:10.3390/nu11030635

Choi, W. J., Dong, H. J., Jeong, H. U., Ryu, D. W., Song, S. M., Kim, Y. R., et al. (2020). Lactobacillus Plantarum LMT1-48 Exerts Anti-obesity Effect in HighFat Diet-Induced Obese Mice by Regulating Expression of Lipogenic Genes. Sci. Rep. 10, 869-9. doi:10.1038/s41598-020-57615-5

Cotillard, A., Kennedy, S. P., Kong, L. C., Prifti, E., Pons, N., Le Chatelier, E., et al. (2013). Dietary Intervention Impact on Gut Microbial Gene Richness. Nature 500, 585-588. doi:10.1038/nature12480
Crawford, P. A., Crowley, J. R., Sambandam, N., Muegge, B. D., Costello, E. K., Hamady, M., et al. (2009). Regulation of Myocardial Ketone Body Metabolism by the Gut Microbiota during Nutrient Deprivation. Proc. Natl. Acad. Sci. US A. 106, 11276-11281. doi:10.1073/pnas.0902366106

De Kort, S., Keszthelyi, D., and Masclee, A. A. (2011). Leaky Gut and Diabetes Mellitus: What Is the Link? Obes. Rev. 12, 449-458. doi:10.1111/j.1467789X.2010.00845.x

Di Cesare, M., Bentham, J., Stevens, G. A., Zhou, B., Danaei, G., Lu, Y., et al. (2016). Trends in Adult Body-Mass index in 200 Countries from 1975 to 2014: a Pooled Analysis of 1698 Population-Based Measurement Studies with 19.2 Million Participants. Lancet 387, 1377-1396. doi:10.1016/S0140-6736(16)30054-X

Eckburg, P. B., Bik, E. M., Bernstein, C. N., Purdom, E., Dethlefsen, L., Sargent, M., et al. (2005). Diversity of the Human Intestinal Microbial flora. Science 308, 1635-1638. doi:10.1126/science.1110591

Ejtahed, H.-S., Angoorani, P., Soroush, A.-R., Atlasi, R., Hasani-Ranjbar, S., Mortazavian, A. M., et al. (2019). Probiotics Supplementation for the Obesity Management; A Systematic Review of Animal Studies and Clinical Trials. J. Funct. Foods 52, 228-242. doi:10.1016/j.jff.2018.10.039

Everard, A., Matamoros, S., Geurts, L., Delzenne, N. M., and Cani, P. D. (2014). Saccharomyces Boulardii Administration Changes Gut Microbiota and Reduces Hepatic Steatosis, Low-Grade Inflammation, and Fat Mass in Obese and Type 2 Diabetic Db/db Mice. MBio 5, e01011-14. doi:10.1128/ mBio.01011-14

Facchin, S., Vitulo, N., Calgaro, M., Buda, A., Romualdi, C., Pohl, D., et al. (2020). Microbiota Changes Induced by Microencapsulated Sodium Butyrate in Patients with Inflammatory Bowel Disease. Neurogastroenterol. Motil. 32, e13914. doi:10.1111/nmo.13914

Fallucca, F., Porrata, C., Fallucca, S., and Pianesi, M. (2014). Influence of Diet on Gut Microbiota, Inflammation and Type 2 Diabetes Mellitus. First Experience with Macrobiotic Ma-Pi 2 Diet. Diabetes Metab. Res. Rev. 30 Suppl 1, 48-54. doi:10.1002/dmrr.2518

Faria, A. M., and Weiner, H. L. (2005). Oral Tolerance. Immunol. Rev. 206, 232-259. doi:10.1111/j.0105-2896.2005.00280.x

Faria, A. M. C., Reis, B. S., and Mucida, D. (2017). Tissue Adaptation: Implications for Gut Immunity and Tolerance. J. Exp. Med. Jem. 214, 1211-1226. doi:10.1084/jem.20162014

Feng, J., Tang, H., Li, M., Pang, X., Wang, L., Zhang, M., et al. (2014). The Abundance of Fecal Faecalibacterium Prausnitzii in Relation to Obesity and Gender in Chinese Adults. Arch. Microbiol. 196, 73-77. doi:10.1007/s00203013-0942-2

Fernandes, J., Su, W., Rahat-Rozenbloom, S., Wolever, T. M., and Comelli, E. M. (2014). Adiposity, Gut Microbiota and Faecal Short Chain Fatty Acids Are Linked in Adult Humans. Nutr. Diabetes 4, e121. doi:10.1038/nutd.2014.23

Fitzgerald, C. B., Shkoporov, A. N., Sutton, T. D. S., Chaplin, A. V., Velayudhan, V., Ross, R. P., et al. (2018). Comparative Analysis of Faecalibacterium Prausnitzii Genomes Shows a High Level of Genome Plasticity and Warrants Separation into New Species-Level Taxa. BMC Genomics 19, 931. doi:10.1186/s12864-0185313-6

Furet, J. P., Kong, L. C., Tap, J., Poitou, C., Basdevant, A., Bouillot, J. L., et al. (2010). Differential Adaptation of Human Gut Microbiota to Bariatric SurgeryInduced Weight Loss: Links with Metabolic and Low-Grade Inflammation Markers. Diabetes 59, 3049-3057. doi:10.2337/db10-0253

Ganesan, K., Chung, S. K., Vanamala, J., and Xu, B. (2018). Causal Relationship between Diet-Induced Gut Microbiota Changes and Diabetes: A Novel Strategy to Transplant Faecalibacterium Prausnitzii in Preventing Diabetes. Int. J. Mol. Sci. 19. 3720. doi:10.3390/ijms19123720

Gibson, G. R., Hutkins, R., Sanders, M. E., Prescott, S. L., Reimer, R. A., Salminen, S. J., et al. (2017). Expert Consensus Document: The International Scientific Association for Probiotics and Prebiotics (ISAPP) Consensus Statement on the Definition and Scope of Prebiotics. Nat. Rev. Gastroenterol. Hepatol. 14, 491-502. doi:10.1038/nrgastro.2017.75

Gómez-Hernández, A., Beneit, N., Díaz-Castroverde, S., and Escribano, Ó. (2016). Differential Role of Adipose Tissues in Obesity and Related Metabolic and Vascular Complications. Int. J. Endocrinol. 2016, 1216783. doi:10.1155/2016/ 1216783

Guerville, M., Leroy, A., Sinquin, A., Laugerette, F., Michalski, M. C., and Boudry, G. (2017). Western-diet Consumption Induces Alteration of Barrier Function Mechanisms in the Ileum that Correlates with Metabolic Endotoxemia in Rats. 
Am. J. Physiol. Endocrinol. Metab. 313, E107-E120. doi:10.1152/ ajpendo.00372.2016

Harig, J. M., Soergel, K. H., Komorowski, R. A., and Wood, C. M. (1989). Treatment of Diversion Colitis with Short-Chain-Fatty Acid Irrigation. N. Engl. J. Med. 320, 23-28. doi:10.1056/NEJM198901053200105

Hippe, B., Remely, M., Aumueller, E., Pointner, A., Magnet, U., and Haslberger, A. G. (2016). Faecalibacterium Prausnitzii Phylotypes in Type Two Diabetic, Obese, and Lean Control Subjects. Benef. Microbes 7, 511-517. doi:10.3920/ BM2015.0075

Ho-Plagaro, A., Santiago-Fernandez, C., García-Serrano, S., Rodriguez, C., Garrido-Sanchez, L., Escamilla, A., et al. (2019). A Lower Duodenal Immune Response Is Associated with an Increase of Insulin Resistance in Patients with Morbid Obesity. Int. J. Obes. 44, 340-352. doi:10.1038/s41366019-0458-1

Hold, G. L., Schwiertz, A., Aminov, R. I., Blaut, M., and Flint, H. J. (2003). Oligonucleotide Probes that Detect Quantitatively Significant Groups of Butyrate-Producing Bacteria in Human Feces. Appl. Environ. Microbiol. 69, 4320-4324. doi:10.1128/AEM.69.7.4320-4324.2003

Hotamisligil, G. S. (2017). Inflammation, Metaflammation and Immunometabolic Disorders. Nature 542, 177-185. doi:10.1038/nature21363

Jo, J., Gavrilova, O., Pack, S., Jou, W., Mullen, S., Sumner, A. E., et al. (2009). Hypertrophy And/or Hyperplasia: Dynamics of Adipose Tissue Growth. Plos Comput. Biol. 5, e1000324. doi:10.1371/journal.pcbi.1000324

Joung, H., Chu, J., Kim, B. K., Choi, I. S., Kim, W., and Park, T. S. (2021). Probiotics Ameliorate Chronic Low-Grade Inflammation and Fat Accumulation with Gut Microbiota Composition Change in Diet-Induced Obese Mice Models. Appl. Microbiol. Biotechnol. 105, 1203-1213. doi:10.1007/s00253-020-11060-6

Kawano, Y., Nakae, J., Watanabe, N., Kikuchi, T., Tateya, S., Tamori, Y., et al. (2016). Colonic Pro-inflammatory Macrophages Cause Insulin Resistance in an Intestinal Ccl2/Ccr2-dependent Manner. Cel Metab 24, 295-310. doi:10.1016/ j.cmet.2016.07.009

Kim, H., Jeong, Y., Kang, S., You, H. J., and Ji, G. E. (2020). Co-Culture with Bifidobacterium Catenulatum Improves the Growth, Gut Colonization, and Butyrate Production of Faecalibacterium Prausnitzii: In Vitro and In Vivo Studies. Microorganisms 8, 788. doi:10.3390/microorganisms8050788

Kim, K. A., Gu, W., Lee, I. A., Joh, E. H., and Kim, D. H. (2012). High Fat DietInduced Gut Microbiota Exacerbates Inflammation and Obesity in Mice via the TLR4 Signaling Pathway. PLoS One 7, e47713. doi:10.1371/ journal.pone.0047713

Kolodziejczyk, A. A., Zheng, D., and Elinav, E. (2019). Diet-microbiota Interactions and Personalized Nutrition. Nat. Rev. Microbiol. 17, 742-753. doi:10.1038/s41579-019-0256-8

Lambertz, J., Weiskirchen, S., Landert, S., and Weiskirchen, R. (2017). Fructose: A Dietary Sugar in Crosstalk with Microbiota Contributing to the Development and Progression of Non-alcoholic Liver Disease. Front. Immunol. 8. doi:10.3389/fimmu.2017.01159

Lewis, J. D., and Abreu, M. T. (2017). Diet as a Trigger or Therapy for Inflammatory Bowel Diseases. Gastroenterology 152, 398-e6. e6. doi:10.1053/j.gastro.2016.10.019

Lopez-Siles, M., Martinez-Medina, M., Surís-Valls, R., Aldeguer, X., Sabat-Mir, M., Duncan, S. H., et al. (2016). Changes in the Abundance of Faecalibacterium Prausnitzii Phylogroups I and II in the Intestinal Mucosa of Inflammatory Bowel Disease and Patients with Colorectal Cancer. Inflamm. Bowel Dis. 22, 28-41. doi:10.1097/MIB.0000000000000590

Luck, H., Tsai, S., Chung, J., Clemente-Casares, X., Ghazarian, M., Revelo, X. S., et al. (2015). Regulation of Obesity-Related Insulin Resistance with Gut Antiinflammatory Agents. Cel Metab 21, 527-542. doi:10.1016/j.cmet.2015.03.001

Lumeng, C. N., Bodzin, J. L., and Saltiel, A. R. (2007). Obesity Induces a Phenotypic Switch in Adipose Tissue Macrophage Polarization. J. Clin. Invest. 117, 175-184. doi:10.1172/JCI29881.The

Madjd, A., Taylor, M. A., Delavari, A., Malekzadeh, R., Macdonald, I. A., and Farshchi, H. R. (2017). Beneficial Effects of Replacing Diet Beverages with Water on Type 2 Diabetic Obese Women Following a Hypo-Energetic Diet: A Randomized, 24-week Clinical Trial. Diabetes Obes. Metab. 19, 125-132. doi:10.1111/dom.12793

Martín, R., Chain, F., Miquel, S., Lu, J., Gratadoux, J. J., Sokol, H., et al. (2014). The Commensal Bacterium Faecalibacterium Prausnitzii Is Protective in
DNBS-Induced Chronic Moderate and Severe Colitis Models. Inflamm. Bowel Dis. 20, 417-430. doi:10.1097/01.MIB.0000440815.76627.64

Martín, R., Miquel, S., Benevides, L., Bridonneau, C., Robert, V., Hudault, S., et al. (2017). Functional Characterization of Novel Faecalibacterium Prausnitzii Strains Isolated from Healthy Volunteers: A Step Forward in the Use of F. Prausnitzii as a Next-Generation Probiotic. Front. Microbiol. 8, 1226. doi:10.3389/fmicb.2017.01226

Martinic, A., Barouei, J., Bendiks, Z., Mishchuk, D., Heeney, D. D., Martin, R., et al. (2018). Supplementation of Lactobacillus Plantarum Improves Markers of Metabolic Dysfunction Induced by a High Fat Diet. J. Proteome Res. 17, 2790-2802. doi:10.1021/acs.jproteome.8b00282

Minami, J., Kondo, S., Yanagisawa, N., Odamaki, T., Xiao, J. Z., Abe, F., et al. (2015). Oral Administration of Bifidobacterium Breve B-3 Modifies Metabolic Functions in Adults with Obese Tendencies in a Randomised Controlled Trial. J. Nutr. Sci. 4, e17. doi:10.1017/jns.2015.5

Miquel, S., Leclerc, M., Martin, R., Chain, F., Lenoir, M., Raguideau, S., et al. (2015). Identification of Metabolic Signatures Linked to Anti-inflammatory Effects of Faecalibacterium Prausnitzii. MBio 6, 1-10. doi:10.1128/mBio.00300-15

Miranda, M. C. G., Oliveira, R. P., Torres, L., Aguiar, S. L. F., Pinheiro-Rosa, N., Lemos, L., et al. (2019). Frontline Science: Abnormalities in the Gut Mucosa of Non-obese Diabetic Mice Precede the Onset of Type 1 Diabetes. J. Leukoc. Biol. 106, 513-529. doi:10.1002/JLB.3HI0119-024RR

Miyamoto, J., Hasegawa, S., Kasubuchi, M., Ichimura, A., Nakajima, A., and Kimura, I. (2016). Nutritional Signaling via Free Fatty Acid Receptors. Int. J. Mol. Sci. 17 (4), 450. doi:10.3390/ijms17040450

Monteiro-Sepulveda, M., Touch, S., Mendes-Sá, C., André, S., Poitou, C., Allatif, O., et al. (2015). Jejunal T Cell Inflammation in Human Obesity Correlates with Decreased Enterocyte Insulin Signaling. Cel Metab 22, 113-124. doi:10.1016/ j.cmet.2015.05.020

Mörkl, S., Lackner, S., Meinitzer, A., Mangge, H., Lehofer, M., Halwachs, B., et al. (2018). Gut Microbiota, Dietary Intakes and Intestinal Permeability Reflected by Serum Zonulin in Women. Eur. J. Nutr. 57, 2985-2997. doi:10.1007/s00394018-1784-0

Munukka, E., Rintala, A., Toivonen, R., Nylund, M., Yang, B., Takanen, A., et al. (2017). Faecalibacterium Prausnitzii Treatment Improves Hepatic Health and Reduces Adipose Tissue Inflammation in High-Fat Fed Mice. ISME J. 11, 1667-1679. doi:10.1038/ismej.2017.24

Navab-Moghadam, F., Sedighi, M., Khamseh, M. E., Alaei-Shahmiri, F., Talebi, M., Razavi, S., et al. (2017). The Association of Type II Diabetes with Gut Microbiota Composition. Microb. Pathog. 110, 630-636. doi:10.1016/ j.micpath.2017.07.034

Nishimura, S., Manabe, I., Nagasaki, M., Eto, K., Yamashita, H., Ohsugi, M., et al. (2009). CD8+ Effector T Cells Contribute to Macrophage Recruitment and Adipose Tissue Inflammation in Obesity. Nat. Med. 15, 914-920. doi:10.1038/ nm.1964

Okubo, T., Takemura, N., Yoshida, A., and Sonoyama, K. (2013). KK/Ta Mice Administered Lactobacillus Plantarum Strain No. 14 Have Lower Adiposity and Higher Insulin Sensitivity. Biosci. Microbiota Food Health 32, 93-100. doi:10.12938/bmfh.32.93

Park, D. Y., Ahn, Y. T., Park, S. H., Huh, C. S., Yoo, S. R., Yu, R., et al. (2013). Supplementation of Lactobacillus Curvatus HY7601 and Lactobacillus Plantarum KY1032 in Diet-Induced Obese Mice Is Associated with Gut Microbial Changes and Reduction in Obesity. PLoS One 8, e59470. doi:10.1371/journal.pone.0059470

Parnell, J. A., Raman, M., Rioux, K. P., and Reimer, R. A. (2012). The Potential Role of Prebiotic Fibre for Treatment and Management of Non-alcoholic Fatty Liver Disease and Associated Obesity and Insulin Resistance. Liver Int. 32, 701-711. doi:10.1111/j.1478-3231.2011.02730.x

Pedersen, H. K., Gudmundsdottir, V., Nielsen, H. B., Hyotylainen, T., Nielsen, T., Jensen, B. A., et al. (2016). Human Gut Microbes Impact Host Serum Metabolome and Insulin Sensitivity. Nature 535, 376-381. doi:10.1038/ nature 18646

Peng, L., He, Z., Chen, W., Holzman, I. R., and Lin, J. (2007). Effects of Butyrate on Intestinal Barrier Function in a Caco-2 Cell Monolayer Model of Intestinal Barrier. Pediatr. Res. 61, 37-41. doi:10.1203/01.pdr.0000250014.92242.f3

Pinto, E., Anselmo, M., Calha, M., Bottrill, A., Duarte, I., Andrew, P. W., et al. (2017). The Intestinal Proteome of Diabetic and Control Children Is Enriched 
with Different Microbial and Host Proteins. Microbiology (Reading) 163, 161-174. doi:10.1099/mic.0.000412

Qin, J., Li, R., Raes, J., Arumugam, M., Burgdorf, K. S., Manichanh, C., et al. (2010). A Human Gut Microbial Gene Catalogue Established by Metagenomic Sequencing. Nature 464, 59-65. doi:10.1038/nature08821

Quévrain, E., Maubert, M. A., Michon, C., Chain, F., Marquant, R., Tailhades, J., et al. (2016). Identification of an Anti-inflammatory Protein from Faecalibacterium Prausnitzii, a Commensal Bacterium Deficient in Crohn's Disease. Gut 65, 415-425. doi:10.1136/gutjnl-2014-307649

Régnier, M., Van Hul, M., Knauf, C., and Cani, P. D. (2021). Gut Microbiome, Endocrine Control of Gut Barrier Function and Metabolic Diseases. J. Endocrinol. 248, R67-R82. doi:10.1530/JOE-20-0473

Respondek, F., Gerard, P., Bossis, M., Boschat, L., Bruneau, A., Rabot, S., et al. (2013). Short-Chain Fructo-Oligosaccharides Modulate Intestinal Microbiota and Metabolic Parameters of Humanized Gnotobiotic Diet Induced Obesity Mice. PLoS One 8, e71026. doi:10.1371/journal.pone.0071026

Rezende, R. M., and Weiner, H. L. (2017). History and Mechanisms of Oral Tolerance. Semin. Immunol. 30, 3-11. doi:10.1016/j.smim.2017.07.004

Rivière, A., Selak, M., Lantin, D., Leroy, F., and De Vuyst, L. (2016). Bifidobacteria and Butyrate-Producing colon Bacteria: Importance and Strategies for Their Stimulation in the Human Gut. Front. Microbiol. 7, 979. doi:10.3389/ fmicb.2016.00979

Rocha, V. Z., Folco, E. J., Ozdemir, C., Sheikine, Y., Christen, T., Sukhova, G. K., et al. (2014). CXCR3 Controls T-Cell Accumulation in Fat Inflammation. Arterioscler. Thromb. Vasc. Biol. 34, 1374-1381. doi:10.1161/ ATVBAHA.113.303133

Rossi, O., Khan, M. T., Schwarzer, M., Hudcovic, T., Srutkova, D., Duncan, S. H., et al. (2015). Faecalibacterium Prausnitzii Strain HTF-F and its Extracellular Polymeric Matrix Attenuate Clinical Parameters in DSS-Induced Colitis. PLoS One 10, e0123013. doi:10.1371/journal.pone.0123013

Rossi, O., Van Berkel, L. A., Chain, F., Tanweer Khan, M., Taverne, N., Sokol, H., et al. (2016). Faecalibacterium Prausnitzii A2-165 Has a High Capacity to Induce IL-10 in Human and Murine Dendritic Cells and Modulates T Cell Responses. Sci. Rep. 6, 18507-18512. doi:10.1038/srep18507

Rush, E. C., Patel, M., Plank, L. D., and Ferguson, L. R. (2002). Kiwifruit Promotes Laxation in the Elderly. Asia Pac. J. Clin. Nutr. 11, 164-168. doi:10.1046/j.14406047.2002.00287.x

Sanders, M. E., Merenstein, D. J., Reid, G., Gibson, G. R., and Rastall, R. A. (2019). Probiotics and Prebiotics in Intestinal Health and Disease: from Biology to the Clinic. Nat. Rev. Gastroenterol. Hepatol. 16, 605-616. doi:10.1038/s41575-0190173-3

Scheppach, W. (1996). Treatment of Distal Ulcerative Colitis with Short-Chain Fatty Acid Enemas. A Placebo-Controlled Trial. German-Austrian SCFA Study Group. Dig. Dis. Sci. 41, 2254-2259. doi:10.1007/BF02071409

Shirvani-Rad, S., Tabatabaei-Malazy, O., Mohseni, S., Hasani-Ranjbar, S., Soroush, A.-R., Hoseini-Tavassol, Z., et al. (2021). Probiotics as a Complementary Therapy for Management of Obesity: A Systematic Review. Evidence-Based Complement. Altern. Med. 2021, 1-11. doi:10.1155/2021/6688450

Simon, M. C., Reinbeck, A. L., Wessel, C., Heindirk, J., Jelenik, T., Kaul, K., et al. (2020). Distinct Alterations of Gut Morphology and Microbiota Characterize Accelerated Diabetes Onset in Nonobese Diabetic Mice. J. Biol. Chem. 295, 969-980. doi:10.1074/jbc.RA119.010816

Sokol, H., Pigneur, B., Watterlot, L., Lakhdari, O., Bermúdez-Humarán, L. G., Gratadoux, J. J., et al. (2008). Faecalibacterium Prausnitzii Is an Antiinflammatory Commensal Bacterium Identified by Gut Microbiota Analysis of Crohn Disease Patients. Proc. Natl. Acad. Sci. U. S. A. 105, 16731-16736. doi:10.1073/pnas.0804812105

Spiekermann, G. M., and Walker, W. A. (2001). Oral Tolerance and its Role in Clinical Disease. J. Pediatr. Gastroenterol. Nutr. 32, 237-255. doi:10.1097/ 00005176-200103000-00003

Sun, K., Kusminski, C. M., and Scherer, P. E. (2011). Adipose Tissue Remodeling and Obesity. J. Clin. Invest. 121, 2094-1012101. Available at: http://www.ncbi. nlm.nih.gov/pubmed/21633177 (Accessed May 31, 2019). doi:10.1172/ JCI45887

Sun, M., Wu, W., Chen, L., Yang, W., Huang, X., Ma, C., et al. (2018). Microbiotaderived Short-Chain Fatty Acids Promote Th1 Cell IL-10 Production to Maintain Intestinal Homeostasis. Nat. Commun. 9, 3555. doi:10.1038/ s41467-018-05901-2
Sun, M., Wu, W., Liu, Z., and Cong, Y. (2017). Microbiota Metabolite Short Chain Fatty Acids, GPCR, and Inflammatory Bowel Diseases. J. Gastroenterol. 52, 1-8. doi:10.1007/s00535-016-1242-9

Talukdar, D., Seenivasan, S., Cameron, A. J., and Sacks, G. (2020). The Association between National Income and Adult Obesity Prevalence: Empirical Insights into Temporal Patterns and Moderators of the Association Using 40 Years of Data across 147 Countries. PLoS One 15, e0232236. doi:10.1371/ journal.pone. 0232236

Thaiss, C. A., Levy, M., Grosheva, I., Zheng, D., Soffer, E., Blacher, E., et al. (2018). Hyperglycemia Drives Intestinal Barrier Dysfunction and Risk for Enteric Infection. Science 359, 1376-1383. doi:10.1126/science.aar3318

Tomé-Castro, X. M., Rodriguez-Arrastia, M., Cardona, D., Rueda-Ruzafa, L., Molina-Torres, G., and Roman, P. (2021). Probiotics as a Therapeutic Strategy in Obesity and Overweight: A Systematic Review. Benef. Microbes 12, 5-15. doi:10.3920/BM2020.0111

Turnbaugh, P. J., Bäckhed, F., Fulton, L., and Gordon, J. I. (2008). DietInduced Obesity Is Linked to Marked but Reversible Alterations in the Mouse Distal Gut Microbiome. Cell Host Microbe 3, 213-223. doi:10.1016/j.chom.2008.02.015

Turnbaugh, P. J., Hamady, M., Yatsunenko, T., Cantarel, B. L., Duncan, A., Ley, R. E., et al. (2009). A Core Gut Microbiome in Obese and Lean Twins. Nature 457, 480-484. doi:10.1038/nature07540

Turnbaugh, P. J., Ley, R. E., Mahowald, M. A., Magrini, V., Mardis, E. R., and Gordon, J. I. (2006). An Obesity-Associated Gut Microbiome with Increased Capacity for Energy Harvest. Nature 444, 1027-1031. doi:10.1038/nature05414

Vancamelbeke, M., Laeremans, T., Vanhove, W., Arnauts, K., Ramalho, A. S., Farré, R., et al. (2019). Butyrate Does Not Protect against InflammationInduced Loss of Epithelial Barrier Function and Cytokine Production in Primary Cell Monolayers from Patients with Ulcerative Colitis. J. Crohns Colitis 13, 1351-1361. doi:10.1093/ecco-jcc/jjz064

Verdam, F. J., Fuentes, S., De Jonge, C., Zoetendal, E. G., Erbil, R., Greve, J. W., et al. (2013). Human Intestinal Microbiota Composition Is Associated with Local and Systemic Inflammation in Obesity. Obesity (Silver Spring) 21, E607-E615. doi:10.1002/oby.20466

Verhoog, S., Taneri, P. E., Roa Díaz, Z. M., Marques-Vidal, P., Troup, J. P., Bally, L., et al. (2019). Dietary Factors and Modulation of Bacteria Strains of Akkermansia Muciniphila and Faecalibacterium Prausnitzii: A Systematic Review. Nutrients 11. doi:10.3390/nu11071565

Vijay-Kumar, M., Aitken, J. D., Carvalho, F. A., Cullender, T. C., Mwangi, S., Srinivasan, S., et al. (2010). Metabolic Syndrome and Altered Gut Microbiota in Mice Lacking Toll-like Receptor 5. Science 328, 228-231. doi:10.1126/ science.1179721

Winer, D. A., Luck, H., Tsai, S., and Winer, S. (2016). The Intestinal Immune System in Obesity and Insulin Resistance. Cel Metab 23, 413-426. doi:10.1016/ j.cmet.2016.01.003

Wolfgang, S., Hartmut, S., Thomas, K., Gian-Maria, P., Peter, B., Stefan, C., et al. (1992). Effect of Butyrate Enemas on the Colonic Mucosa in Distal Ulcerative Colitis. Gastroenterology 103, 51-56. doi:10.1016/00165085(92)91094-K

Wrzosek, L., Ciocan, D., Borentain, P., Spatz, M., Puchois, V., Hugot, C., et al. (2018). Transplantation of Human Microbiota into Conventional Mice Durably Reshapes the Gut Microbiota. Sci. Rep. 8, 6854. doi:10.1038/s41598-01825300-3

Xu, J., Liang, R., Zhang, W., Tian, K., Li, J., Chen, X., et al. (2019). Faecalibacterium Prausnitzii -derived Microbial Anti-inflammatory Molecule Regulates Intestinal Integrity in Diabetes Mellitus Mice via Modulating Tight junction Protein Expression. J. Diabetes 12, 224-236. doi:10.1111/1753-0407.12986

Yang, H., Youm, Y. H., Vandanmagsar, B., Rood, J., Kumar, K. G., Butler, A. A., et al. (2009). Obesity Accelerates Thymic Aging. Blood 114, 3803-3812. doi:10.1182/blood-2009-03-213595

Yoo, S. R., Kim, Y. J., Park, D. Y., Jung, U. J., Jeon, S. M., Ahn, Y. T., et al. (2013). Probiotics L. Plantarum and L. Curvatus in Combination Alter Hepatic Lipid Metabolism and Suppress Diet-Induced Obesity. Obesity (Silver Spring) 21, 2571-2578. doi:10.1002/oby.20428

Zhang, J., Huang, Y.-J., Yoon, J. Y., Kemmitt, J., Wright, C., Schneider, K., et al. (2021). Primary Human Colonic Mucosal Barrier Crosstalk with Super 
Oxygen-Sensitive Faecalibacterium Prausnitzii in Continuous Culture. Med 2, 74-98. e9. doi:10.1016/j.medj.2020.07.001

Zhang, X., Shen, D., Fang, Z., Jie, Z., Qiu, X., Zhang, C., et al. (2013). Human Gut Microbiota Changes Reveal the Progression of Glucose Intolerance. PLoS One 8, e71108. doi:10.1371/journal.pone.0071108

Zhao, X., Higashikawa, F., Noda, M., Kawamura, Y., Matoba, Y., Kumagai, T., et al. (2012). The Obesity and Fatty Liver Are Reduced by Plant-Derived Pediococcus Pentosaceus LP28 in High Fat Diet-Induced Obese Mice. PLoS One 7, e30696. doi:10.1371/journal.pone.0030696

Zhou, D., Pan, Q., Xin, F. Z., Zhang, R. N., He, C. X., Chen, G. Y., et al. (2017). Sodium Butyrate Attenuates High-Fat Diet-Induced Steatohepatitis in Mice by Improving Gut Microbiota and Gastrointestinal Barrier. World J. Gastroenterol. 23, 60-75. doi:10.3748/wjg.v23.i1.60

Zhou, L., Zhang, M., Wang, Y., Dorfman, R. G., Liu, H., Yu, T., et al. (2018). Faecalibacterium Prausnitzii Produces Butyrate to Maintain Th17/Treg Balance and to Ameliorate Colorectal Colitis by Inhibiting Histone Deacetylase 1. Inflamm. Bowel Dis. 24, 1926-1940. doi:10.1093/ibd/izy182
Conflict of Interest: The authors declare that the research was conducted in the absence of any commercial or financial relationships that could be construed as a potential conflict of interest.

Publisher's Note: All claims expressed in this article are solely those of the authors and do not necessarily represent those of their affiliated organizations, or those of the publisher, the editors and the reviewers. Any product that may be evaluated in this article, or claim that may be made by its manufacturer, is not guaranteed or endorsed by the publisher.

Copyright @ 2021 Maioli, Borras-Nogues, Torres, Barbosa, Martins, Langella, Azevedo and Chatel. This is an open-access article distributed under the terms of the Creative Commons Attribution License (CC BY). The use, distribution or reproduction in other forums is permitted, provided the original author(s) and the copyright owner(s) are credited and that the original publication in this journal is cited, in accordance with accepted academic practice. No use, distribution or reproduction is permitted which does not comply with these terms. 